PROCEEDINGS OF THE

AMERICAN MATHEMATICAL SOCIETY

Volume 136, Number 7, July 2008, Pages 2279-2288

S 0002-9939(08)09017-5

Article electronically published on March 13, 2008

\title{
AN ALGEBRAIC EXTENSION OF THE MACMAHON MASTER THEOREM
}

PAVEL ETINGOF AND IGOR PAK

(Communicated by Jim Haglund)

\begin{abstract}
We present a new algebraic extension of the classical MacMahon Master Theorem. The basis of our extension is the Koszul duality for nonquadratic algebras defined by Berger. Combinatorial implications are also discussed.
\end{abstract}

\section{INTRODUCTION}

The MacMahon Master Theorem is a fundamental result in Enumerative Combinatorics, with a number of applications to partition theory, binomial identities and q-series. Ever since Percy MacMahon introduced it in [MM], the theorem was understood largely in a combinatorial and analytic context (see [CF, G.J]). In an important development, a quantum analogue was discovered by Garoufalidis, Lê and Zeilberger [GLZ], sharply extending a basic partially commutative version of Cartier and Foata $[\mathrm{CF}$. Since then, several other algebraic extensions have been introduced [FH2, [KP], but their nature remained mysterious.

Most recently, Hai and Lorenz showed that the quantum analogue can be obtained from the Koszul duality HL. While $\mathrm{HL}$ does not rederive the results of other extensions (which appeared independently), it goes a long way to explain them, in particular the fact that all these algebraic extensions are based on quadratic algebras with Koszul property. It is a natural question whether further (non-quadratic) extensions can be obtained.

In this paper we present an extension of the MacMahon Master Theorem of a new type. The construction is based on special non-commutative algebras $\mathcal{B}_{m, k}$ studied by Berger in $[\mathrm{B}$. These algebras have $m$ generators and homogenous relations of degree $k$ coming from the exterior algebra. Algebras $\mathcal{B}_{m, k}$ are fundamental examples of the generalized Koszul property for non-quadratic algebras defined in [B]. Our proof uses the Hai-Lorenz approach and Berger's explicit calculations of the Koszul resolutions in this case. The result is a curious algebraic identity which can be stated in a combinatorial language.

In this note, we first recall the MacMahon Master Theorem and state the main result in Section 1. We then give a combinatorial interpretation in Section 2. Two natural examples and enumerative applications are considered in Sections 3, 4, while the proofs are postponed until Section 5 . We conclude with final remarks and open problems (Section 6).

Received by the editors August 1, 2006.

2000 Mathematics Subject Classification. Primary 16S37; Secondary 05A30, 05E05, 81S05.

(C)2008 American Mathematical Society 2279

Reverts to public domain 28 years from publication 
In conclusion, let us say a few words about the fundamental difference between an algebraic and a combinatorial approach to the Master Theorem. A combinatorial approach starts by translating the algebraic identity as an equation for two sums of words in a formal language. This equation is then proved by an explicit bijection. The algebraic approach starts by showing that both sides of the algebraic identity are invariant under a large group of symmetries. This action is then utilized to obtain a concise and insightful proof of the result. The main difference is that in the combinatorial approach the bijection itself is of value, and its properties often lead to new results. We refer to $\mathrm{CF}, \mathrm{FZ}, \mathrm{KP}$. for examples of this phenomenon in the context of the MacMahon Master Theorem. It would be interesting to obtain a combinatorial proof of our main result (Theorem 21), and further extend it in these directions.

\section{MAIN RESUlts}

We begin by stating the MacMahon Master Theorem in the classical form:

Theorem 1 (MacMahon Master Theorem). Let $A=\left(a_{i j}\right)_{m \times m}$ be a complex matrix, and let $x_{1}, \ldots, x_{m}$ be a set of variables. Denote by $G\left(k_{1}, \ldots, k_{m}\right)$ the coefficient of $x_{1}^{k_{1}} \cdots x_{m}^{k_{m}}$ in

$$
\prod_{i=1}^{m}\left(a_{i 1} x_{1}+\ldots+a_{i m} x_{m}\right)^{k_{i}} .
$$

Let $t_{1}, \ldots, t_{m}$ be another set of variables, and $T=\left(\delta_{i j} t_{i}\right)_{m \times m}$. Then

$$
\sum_{\left(k_{1}, \ldots, k_{m}\right)} G\left(k_{1}, \ldots, k_{m}\right) t_{1}^{k_{1}} \cdots t_{m}^{k_{m}}=\frac{1}{\operatorname{det}(I-T A)},
$$

where the summation is over all non-negative integer vectors $\left(k_{1}, \ldots, k_{m}\right)$.

By taking $t_{1}=\ldots=t_{m}=1$ we get

$$
\sum_{\left(k_{1}, \ldots, k_{m}\right)} G\left(k_{1}, \ldots, k_{m}\right)=\frac{1}{\operatorname{det}(I-A)},
$$

whenever both sides of the equation are well defined. It is easy to see that (2) and (3) hold when all $a_{i j}$ are formal commutative variables. Moreover, replacing $a_{i j}$ in (3) with $a_{i j} t_{i}$ shows that (3) is equivalent to (2), so in the literature the MacMahon Master Theorem is often stated in this form.

Let us now move into a non-commutative setting. Fix an integer $k, 2 \leq k \leq m$. Let $\mathcal{B}=\mathcal{B}_{m, k}$ be an algebra with basis $X=\left\{x_{1}, \ldots, x_{m}\right\}$ and the following defining relations:

$$
\sum_{\sigma \in S_{k}}(-1)^{\operatorname{sign}(\sigma)} x_{i_{\sigma(1)}} \cdots x_{i_{\sigma(k)}}=0
$$

for all $1 \leq i_{1}<\ldots<i_{k} \leq m$. When $k=2$ we get the usual symmetric algebra $\mathbb{C}\left[x_{1}, \ldots, x_{m}\right]$. Define the set $\Lambda(m, k)$ of admissible sequences $\mathbf{i}=\left(i_{1} \ldots i_{\ell}\right)$, where $i_{1}, \ldots, i_{\ell} \in\{1, \ldots, m\}$, and such that no $k$ subsequent indices are strictly decreasing. We call monomials

$$
M_{\mathbf{i}}(X)=x_{i_{1}} \cdots x_{i_{\ell}}, \quad \text { where } \mathbf{i}=\left(i_{1}, \ldots, i_{\ell}\right) \in \Lambda(m, k),
$$

the admissible monomials. It is known (see [B, p. 723]) and easy to see that the set of admissible monomials $M_{\mathbf{i}}(X)$ is a linear basis in $\mathcal{B}$ (use a Gröbner basis 
argument with the lexicographic ordering). When $k=2$ we get the usual basis in $\mathbb{C}\left[x_{1}, \ldots, x_{m}\right]$ with indices $\left(i_{1}, \ldots, i_{\ell}\right) \in \Lambda(m, 2)$ weakly increasing.

Now fix a matrix $A=\left(a_{i j}\right), a_{i j} \in \mathbb{C}$, and let

$$
M_{\mathbf{i}}(A X)=y_{i_{1}} \cdots y_{i_{\ell}} \text {, where } y_{i}=a_{i 1} x_{1}+\ldots+a_{i m} x_{m} \text {, for all } 1 \leq i \leq m .
$$

The product $M_{\mathbf{i}}(A X)$ is an element in algebra $\mathcal{B}$ and is a generalization of the product (11). For an admissible sequence $\mathbf{i}=\left(i_{1}, \ldots, i_{\ell}\right) \in \Lambda(m, k)$, denote by $G(\mathbf{i})=G\left(i_{1}, \ldots, i_{\ell}\right)$ the coefficient of $M_{\mathbf{i}}(X)$ in $M_{\mathbf{i}}(A X)$. We set $G(\mathbf{i})=1$ for an empty sequence $\mathbf{i}=\varnothing$.

Theorem 2. Let $A=\left(a_{i j}\right)_{m \times m}$ be a complex matrix, let $t_{1}, \ldots, t_{m}$ be another set of variables, which commute with $x_{i}$ and each other, and let $T=\left(\delta_{i j} t_{i}\right)_{m \times m}$. Then

$$
\sum_{\left(i_{1}, \ldots, i_{\ell}\right) \in \Lambda(m, k)} G\left(i_{1}, \ldots, i_{\ell}\right) t_{i_{1}} \cdots t_{i_{\ell}} \cdot \sum_{r=0,1 \bmod k}(-1)^{\alpha(r)} c_{r}(T A)=1
$$

where $\alpha(r)=r-(r \bmod k)$, and polynomials $c_{i}(T A) \in \mathbb{C}\left[t_{1}, \ldots, t_{m}\right]$ are defined as coefficients of the characteristic polynomial:

$$
\operatorname{det}(\lambda I-T A)=\sum_{r=0}^{m} c_{r}(T A) \lambda^{m-r} .
$$

Note that when $k=2$ the power $\alpha(r)$ is always even, and $r$ takes all non-negative values $\leq m$. Therefore, the second sum in (5) is equal to $\operatorname{det}(I-T A)$, as in (2). Similarly, each term in the first summation in (5) is equal to the corresponding term in (2). This implies that Theorem 2 is an extension of the MacMahon Master Theorem 1

\section{Combinatorial Reformulation}

In this section we restate our main result, Theorem 2 in the language of words. We assume that $a_{i j}, 1 \leq i, j \leq m$, are commutative variables. We begin with some combinatorial definitions.

Denote $[m]=\{1, \ldots, m\}$ and let $\mathbf{i}=\left(i_{1}, \ldots, i_{\ell}\right) \in[m]^{\ell}$. Recall that $\Lambda(m, k)$ denotes the set of admissible sequences $\mathbf{i}$. Denote by $\Lambda(m, k, \ell)$ the set of sequences of length $\ell$. Let

$$
\operatorname{inv}(\mathbf{i})=\left|\left\{(s, t): i_{s}>i_{t}, s<t\right\}\right|
$$

be the (usual) number of inversion in sequence $\mathbf{i}$. Define the smallest decreasing $k$ sequence in $\mathbf{i}$ to be a subsequence $i_{s}>i_{s+1}>\ldots>i_{s+k-1}$, where $s$ is the smallest possible.

We say that two sequences $\mathbf{i}, \mathbf{i}^{\prime} \in[m]^{\ell}$ differ by a $k$-reversion, and write $\mathbf{i} \rightarrow$ $\mathbf{i}^{\prime}$, if $\mathbf{i}$ differs from $\mathbf{i}^{\prime}$ by a permutation of elements of the smallest decreasing $k$ sequence in $\mathbf{i}^{\prime}$. Of course, $\operatorname{inv}\left(\mathbf{i}^{\prime}\right)>\operatorname{inv}(\mathbf{i})$ in this case. For example, $(4,3,2,6,1) \rightarrow$ $(4,6,3,2,1)$, where $k=3, m=6, \ell=5$, and $(6,3,2)$ is the smallest decreasing 3 -sequence in $(4,6,3,2,1) \in[6]^{5}$. Here $\operatorname{inv}(4,3,2,6,1)=7<\operatorname{inv}(4,6,3,2,1)=9$.

By a reversion path from $\mathbf{i}$ to $\mathbf{j}$, where $\mathbf{i}, \mathbf{j} \in[m]^{\ell}$, we call a sequence of $k$ reversions:

$$
\gamma: \mathbf{i} \rightarrow \mathbf{i}^{\prime} \rightarrow \mathbf{i}^{\prime \prime} \rightarrow \ldots \rightarrow \mathbf{j}
$$

We write $\mathbf{i} \rightarrow_{\gamma} \mathbf{j}$ in this case, and let $|\gamma|$ denote length (number of $k$-reversions) in path $\gamma$. Since at every $k$-reversion the number of inversion increases, we clearly 
have $\operatorname{inv}(\mathbf{j})-\operatorname{inv}(\mathbf{i}) \geq|\gamma|$. Define integer coefficients

$$
c_{\mathbf{i}, \mathbf{j}}=\sum_{\mathbf{i} \rightarrow \gamma \mathbf{j}}(-1)^{\operatorname{inv}(\mathbf{j})-\operatorname{inv}(\mathbf{i})-|\gamma|},
$$

where the summation is over all reversion paths $\gamma$ from $\mathbf{i}$ to $\mathbf{j}$. Note that when $k=2$, we have $c_{\mathbf{i}, \mathbf{j}} \in\{0,1\}$ depending on whether $\mathbf{j} \succ \mathbf{i}$ in the weak Bruhat order. Let us consider the products

$$
a_{\mathbf{i j}}=a_{i_{1} j_{1}} \cdots a_{i_{\ell} j_{\ell}},
$$

where $\mathbf{i}=\left(i_{1}, \ldots, i_{\ell}\right), \mathbf{j}=\left(j_{1}, \ldots, j_{\ell}\right) \in[m]^{\ell}$.

A partial permutation $\omega$ of $[m]$ is defined as a pair $J \subset[m]$ and the usual permutation of $J$ (which we also denote by $\omega$ ). By $\operatorname{inv}(\omega)$ denote the (usual) number of inversion of the permutations of elements in $J$. Denote by $\Sigma_{m}(r)$ the set of partial permutations of $[m]$, such that $|J|=r$. For a partial permutation $\omega \in \Sigma_{m}(r)$ define

$$
a_{\omega}=a_{j_{1} \omega\left(j_{1}\right)} \cdots a_{j_{r} \omega\left(j_{r}\right)},
$$

where $J=\left\{j_{1}, \ldots, j_{r}\right\}$. Set $a_{\omega}=1$ for an empty partial permutation (when $J=\varnothing$ ).

Corollary 3. For $k \leq m$, in notation above we have:

$$
\begin{aligned}
& {\left[1+\sum_{\ell=1}^{\infty} \sum_{\mathbf{i} \in \Lambda(m, k, \ell)} \sum_{\mathbf{j} \in[m]^{\ell}} c_{\mathbf{i}, \mathbf{j}} \cdot a_{\mathbf{i j}}\right]} \\
& \cdot\left[\sum_{r=0,1 \bmod k}(-1)^{\alpha(r)} \sum_{\omega \in \Sigma_{m}(r)}(-1)^{\operatorname{inv}(\omega)} a_{\omega}\right]=1,
\end{aligned}
$$

where $\alpha(r)=r-(r \bmod k)$.

Let us emphasize that the first term in (5) is an infinite sum. In contrast with the case $k=2$, it no longer has only positive coefficients. On the other hand, the second term is a finite summation, and the sign pattern is completely prescribed.

In fact, the corollary is equivalent to Theorem 2, This follows from the proof of the corollary given in Section 5 .

\section{First exAmple}

Let $A=\left(a_{i j}\right)=I$ be the identity matrix. Then $M_{\mathbf{i}}(A X)=M_{\mathbf{i}}(X)$ and $G(\mathbf{i})=1$, for all $\mathbf{i} \in[m]^{\ell}$. Therefore, the first term in (5) is equal to the weighted sum over all admissible sequences, which we denote by $F_{m, k}$ :

$$
F_{m, k}\left(t_{1}, \ldots, t_{m}\right)=\sum_{\left(i_{1}, \ldots, i_{\ell}\right) \in \Lambda(m, k, \ell)} t_{i_{1}} \cdots t_{i_{\ell}} .
$$

For the second term, note that

$$
\operatorname{det}(\lambda I-T A)=\left(\lambda-t_{1}\right) \cdots\left(\lambda-t_{m}\right) .
$$

Therefore, the coefficients of the characteristic polynomial defined in (6) are equal to the elementary symmetric polynomials: $c_{i}=(-1)^{i} e_{i}\left(t_{1}, \ldots, t_{i}\right)$. Now equation (5) gives a closed formula for the infinite summation $F_{m, k}$ :

$$
F_{m, k}=\frac{1}{1-e_{1}+e_{k}-e_{k+1}+e_{2 k}-e_{2 k+1}+\ldots},
$$


where indices in the denominator are $\leq m$. In particular, this implies that $F_{m, k}$ is a symmetric function (see Section 6 for further remarks). Of course, when $k=2$, equation (9) is the classical symmetric functions identity connecting complete and elementary symmetric functions:

$$
1+h_{1}+h_{2}+h_{3}+\ldots=\frac{1}{1-e_{1}+e_{2}-e_{3}+\ldots+(-1)^{m} e_{m}} .
$$

We refer to $[\mathrm{Ma},[\mathrm{S}]$ for the background, proofs and other results on symmetric functions. In the opposite extreme, when $k=m$, we have:

$$
F_{m, m}\left(t_{1}, \ldots, t_{m}\right)=\frac{1}{1-\left(t_{1}+\ldots+t_{m}\right)+t_{1} \cdots t_{m}} .
$$

This equation can be shown directly by a simple inductive argument.

Now consider the enumerative applications of our equations. Denote by $L_{m, k}(\ell)$ $=|\Lambda(m, k, \ell)|$ the number of admissible sequences. Taking $t_{1}=\ldots=t_{m}=t$ in (9) we obtain a generating function for the number of admissible sequences:

$$
1+\sum_{\ell=1}^{\infty} L_{m, k}(\ell) t^{\ell}=\left(1-m t+\left(\begin{array}{c}
m \\
k
\end{array}\right) t^{k}-\left(\begin{array}{c}
m \\
k+1
\end{array}\right) t^{k+1}+\ldots\right)^{-1} .
$$

While the exact shape of (12) may seem surprising, the fact that it is a rational function is not. To see this, consider a graph $\Gamma_{m, k}$ on $\Lambda(m, k, m)$ with (directed) edges of the type:

$$
\left(i_{1}, i_{2}, \ldots, i_{m}\right) \rightarrow\left(i_{2}, i_{3}, \ldots, i_{m}, j\right)
$$

Clearly, $L_{m, k}(m)=m^{\ell}-\left(\begin{array}{c}m \\ k\end{array}\right)$, since $\left(i_{1}>i_{2}>\ldots>i_{m}\right)$ are the only nonadmissible sequences in this case. Observe that admissible sequences of length $\ell$ correspond to paths of length $(\ell-m+1)$ in $\Gamma_{m}$. Using the transfer matrix method (see [S, Chapter 4]) we obtain a rational generating function for $L_{m, k}(m)$, for any fixed $m \geq k$. Note also that the transfer matrix method gives a denominator of degree $\left|\Gamma_{m}\right| \sim m^{\ell}$, much larger than the degree in (12), where it is $\leq m$.

\section{SeCond EXAmple}

Let $k=m$ and suppose $A=\left(a_{i j}\right)$, where $a_{i j}=1$ for all $i, j \in[m]$. In this special case we have:

$$
M_{\mathbf{i}}(A X)=\left(x_{1}+\ldots+x_{m}\right)^{\ell},
$$

for all $\mathbf{i} \in[m]^{\ell}$. Setting $t_{1}=\ldots=t_{m}=t$ in (5), we obtain the first term:

$$
\sum_{\mathbf{i} \in \Lambda(m, k, \ell)} G(\mathbf{i}) t^{\ell}=1+\sum_{\ell=1}^{\infty} N_{m}(\ell) t^{\ell},
$$

where $N_{m}(\ell)$ is the sum of the coefficients of all admissible monomials in $\left(x_{1}+\ldots+\right.$ $\left.x_{m}\right)^{\ell}$.

Now, the module condition for the second term in (5) is very restrictive: only $r=0, r=1$, and $r=m$ are allowed. There is a unique empty partial permutation corresponding to the $r=0$ case, and exactly $m$ partial permutation corresponding to the $r=1$ case (all consisting of single elements). Theorem 2 now gives:

$$
1+\sum_{\ell=1}^{\infty} N_{m}(\ell) t^{\ell}=\frac{1}{\left|\Sigma_{m}(0)\right|-\left|\Sigma_{m}(1)\right| t+\operatorname{det}(A) t^{m}}=\frac{1}{1-m t} .
$$


We conclude that $N_{m}(\ell)=m^{\ell}$, which may seem quite surprising given that the number $L_{m, m}(\ell)$ of admissible sequences in $\Lambda(m, m, \ell)$ is much smaller than $m^{\ell}$. To see this, recall the generating function from the first example:

$$
1+\sum_{\ell=1}^{\infty} L_{m, m}(\ell) t^{\ell}=\frac{1}{1-m t+t^{m}}
$$

which is a special case of (11). From (13) it follows easily that

$$
L_{m, m}(\ell)=\beta^{\ell}(1+o(1))
$$

as $\ell \rightarrow \infty$, for some $\beta<m$. Of course, this can also be seen by a direct argument.

Now, to explain the identity $N_{m}(\ell)=m^{\ell}$, consider what happens when the relations (44) in algebra $\mathcal{B}_{m, m}$ are applied to the $m^{\ell}$ terms in $\left(x_{1}+\ldots+x_{m}\right)^{\ell}$. We have:

$$
x_{\mathbf{j}}=\sum_{\mathbf{j}^{\prime}}(-1)^{\operatorname{sign}(\cdot)} x_{\mathbf{j}^{\prime}},
$$

where the sum is over $m !-1$ permutations $\mathbf{j}^{\prime}$ of the smallest decreasing subsequence in $\mathbf{j}$ and the sign is the sign of these permutations. Clearly, the total sum of coefficients of terms on the right-hand side of (14) is equal to 1 . Therefore, the sum of coefficients of terms is unchanged under the algebra relations, and thus equal to $m^{\ell}$, the initial sum of all coefficients.

\section{Proof of RESUlts}

Proof of Corollary 3. First, note that we used equation (5) with $t_{1}=\ldots=t_{m}=1$. The second term in (8) can be easily seen to be equal to the second product in (5). This follows from the usual expansion of determinant:

$$
\operatorname{det}(\lambda I-A)=\sum_{r=0}^{m} \sum_{\omega \in \Sigma_{m}(r)} \lambda^{m-r}(-1)^{\operatorname{inv}(\omega)} a_{\omega},
$$

as in (6). Now, to see that the first term in (8) is equal to that in (5) we first observe that

$$
x_{j_{1}} \cdots x_{j_{\ell}}=\sum_{\mathbf{i}=\left(i_{1}, \ldots, i_{\ell}\right) \in \Lambda(m, k, \ell)} c_{\mathbf{i}, \mathbf{j}} x_{i_{1}} \cdots x_{j_{\ell}},
$$

for every $\mathbf{j}=\left(j_{1}, \ldots, j_{\ell}\right) \in[m]^{\ell}$. This follows by induction. Start with a product $x_{j_{1}} \cdots x_{j_{\ell}}$ corresponding to $\mathbf{j}$ and apply the relations in algebra $\mathcal{B}_{m, k}$ to the smallest decreasing $k$-sequence. We obtain products $x_{j_{1}^{\prime}} \cdots x_{j_{\ell}^{\prime}}$ corresponding to $k$-reversions $x_{\mathbf{j}^{\prime}} \rightarrow x_{\mathbf{j}}$. Now apply this relation again, and repeat this until only admissible sequences are obtained. Then each reversion path $\mathbf{i} \rightarrow_{\gamma} \mathbf{j}$ corresponds to a term in the summation. After checking that the signs in (7) correspond to those given by (4), we obtain equation (15).

Note now that $G\left(i_{1}, \ldots, i_{\ell}\right)$, defined as the coefficient of $M_{i_{1}, \ldots, i_{\ell}}(X)$ in $M(A X)$, is equal to the summation of products $a_{\mathbf{i j}}$ with coefficients $c_{\mathbf{i}, \mathbf{j}}$. Checking the terms of the summation, we obtain the result.

Proof of Theorem 2. Before we move to the general case, let us first recall the algebraic proof of the MacMahon Master Theorem (see GLZ, HL]). We rewrite 
equation (3) as follows:

$$
\sum_{\ell=0}^{\infty} \operatorname{Tr}\left(S^{\ell} A\right)=\frac{1}{\operatorname{det}(I-A)}
$$

where $V$ denote the vector space of variables $x_{1}, \ldots, x_{m}$. Here the left-hand side is equal to the left-hand side of (3) by definition of the symmetric power $S^{\ell}(A)$. Now, there is a natural action of $G L_{m}(\mathbb{C})$ on both sides. Since matrices with distinct eigenvalues are dense in all matrices, it suffices to show the result for diagonal matrices. In this case equation (3) is equivalent to equation (2) for $A=I$. Finally, the latter can be written as (10) which can be proved by a straightforward calculation (see e.g. $[\mathrm{Ma}, \underline{\mathrm{S}}$ ).

Let us note here that $S^{\ell} V$ is a homogeneous component of the natural action of $G L(V)$ on the symmetric algebra $\mathcal{A}=\mathbb{C}\left[x_{1}, \ldots, x_{m}\right]$. Then the left-hand side of (2) is exactly the character of the $G L(V)$-action in $\mathcal{A}$. Now Theorem 1 follows immediately from here.

In the general case $k>2$ we can proceed in a similar way and reduce the theorem to the case of diagonal matrices, which in turn reduces to the case $A=I$. As shown in Section 3, this can be written as (91). Unfortunately, the above mentioned straightforward calculation is no longer possible in this case (cf. Section 6.3). Following the idea in $\mathrm{HL}$, we take a different approach.

We use the notion of generalized Koszulity defined in [B]. By Theorem 3.13 of $[\mathrm{B}$, the algebras $\mathcal{B}$ defined in Section 1 are generalized Koszul. We can now proceed with the proof of the theorem.

First, note that it is sufficient to prove the theorem in the case when $A$ is a non-degenerate matrix, since such matrices are dense in the space of all matrices. Thus, we may assume that $A \in G L_{m}(\mathbb{C})=G L(V)$, where $V$ (as before) is the space of generators $x_{1}, \ldots, x_{m}$.

Since the relations of the algebra $\mathcal{B}$ are $G L(V)$-invariant, the homogeneous components of this algebra are representations of $G L(V)$. Therefore, the characters of these representations may be computed from the Koszul resolution (see [Fr, PP] for the background). It follows from [B] that

$$
\operatorname{Tor}_{\mathcal{B}}^{2 j+\varepsilon}(\mathbb{C}, \mathbb{C})=\bigwedge^{k j+\varepsilon} V
$$

sitting in degree $k j+\varepsilon$, where $\varepsilon=0,1$, and where $\bigwedge^{d} V$ denotes the exterior powers. Therefore, the computation of the character via the Koszul resolution yields

$$
\sum_{p \geq 0} \operatorname{Tr}\left(\left.A\right|_{\mathcal{B}[p]}\right) t^{p}=\left(\sum_{j \geq 0} \sum_{\varepsilon=0,1}(-1)^{\varepsilon} \operatorname{Tr}\left(\left.A\right|_{\Lambda^{k j+\varepsilon} V}\right) t^{k j+\varepsilon}\right)^{-1},
$$

where $\mathcal{B}[p]$ denotes the $p$-homogenous component. Recall that the first factor in (5) is exactly the character of the $G L(V)$-action in $\mathcal{B}$. The above equation then implies the theorem.

\section{Final REMARKS}

6.1. There are other examples of algebras with $k$-homogeneous relations which satisfy generalized Koszul property [B]. For example, one can omit all signs in (4) and obtain a result similar to Theorem 2, where the second summation in (15) has 
to be modified by replacing exterior powers with symmetric powers. By analogy with (12) we get the following generating function formula:

$$
\sum_{\left(i_{1}, \ldots, i_{\ell}\right) \in \Lambda^{\prime}(m, k, \ell)} t_{i_{1}} \cdots t_{i_{\ell}}=\frac{1}{1-h_{1}+h_{k}-h_{k+1}+h_{2 k}-h_{2 k+1}+\ldots},
$$

where $\Lambda^{\prime}(m, k, \ell)$ denotes the set of sequences $\left(i_{1}, \ldots, i_{\ell}\right) \in[m]^{\ell}$ without $k$ subsequent elements which are weakly decreasing. When $k=2$ we yet again get (2), up to a substitution $t_{i} \leftarrow\left(-t_{i}\right)$. For $k>2$, both the left-hand side and the denominator in the right-hand side are an infinite series, so the result is somewhat less natural from combinatorial point of view.

Let me mention here the work by Roby $[\mathrm{R}]$ who found an evaluation of (16) in a different form. This formula was later proved combinatorially by Foata 1

6.2. Following [GLZ (see also [FH1, [HL, KP]), there is a natural quantum analogue of Theorem 2. The algebra relations (4) have to be replaced with

$$
\sum_{\sigma \in S_{k}}(-q)^{\left(\begin{array}{c}
k \\
2
\end{array}\right)-\operatorname{inv}(\sigma)} x_{i_{\sigma(1)}} \cdots x_{i_{\sigma(k)}}=0,
$$

where $q \in \mathbb{C}, q \neq 0$. This algebra is a flat deformation of $\mathcal{B}_{m, k}$, and its generalized Koszulity follows from the Gröbner basis argument in Berger's proof for $\mathcal{B}_{m, k}[\mathrm{~B}$. Another difference is in the equation (6) which will now contain $q$-minors. This generalization of Theorem 2 is equivariant under $U_{q}(G L(V))$ and can be proved along the same lines.

One can similarly find $k$-homogenous extensions of the Cartier-Foata Theorem and other recently considered generalizations of the MacMahon Master Theorem (see [FH2, $\mathrm{KP}]$ ).

6.3. Although equations (9) and (16) do not seem to be available in the literature, there are at least two ways in which they can be derived from the much more general known combinatorial results. On one hand, the equations follow from the Maximal String Decomposition Theorem (see [GJ, §4.2]), and on the other hand from the Cluster Theorem (see [GJ, §2.8] and [S, Exc. 4.14]). It would be interesting to find a direct bijective proof of the equations.

As we mentioned in Section 5 equation (9) and the eigenvalue argument give an alternative proof of Theorem 2, a proof which does not use Koszulity. Moreover, one can reverse the argument in the proof and derive Berger's result on the Hilbert series of $B_{m, k}$ from the theorem. In other words, one can now prove Berger's result combinatorially, from the Cluster or MSD theorems.

Finally, let us mention that both Cluster and MSD theorems work in the noncommutative setting. In particular, the second part of the MSD Theorem (as stated in [GJ]) gives a natural $q$-analogue according to the number of inversions in a sequence. We are curious to see the relationship between this $q$-analogue and the quantum analogue described above.

6.4. To see that $F_{m, k}$ is symmetric simply observe that

$$
F_{m, k}\left(x_{1}, \ldots, x_{m}\right)=\sum_{\nu} s_{\nu}\left(x_{1}, \ldots, x_{m}\right),
$$

\footnotetext{
${ }^{1}$ Personal communication.
} 
where the summation is over all rim hook (ribbon) diagrams $\nu$ with all columns smaller than $k$ (see e.g. $\left[\mathrm{Ma}, \mathrm{S}\right.$ ). In this case every Schur polynomials $s_{\nu}$ is a summation over all sequences with a descent set corresponding to $\nu$. Their symmetry follows from the usual considerations.

6.5. Even though equation (12) is a simple evaluation of (9), the closest we could find in the literature is the following formula:

$$
1+\sum_{n=1}^{\infty} E(n) \frac{t^{n}}{n !}=\left(1-x+\frac{x^{k}}{k !}-\frac{x^{k+1}}{(k+1) !}+\ldots\right)^{-1}
$$

where $E(n)$ is the number of permutations in $S_{n}$ with no decreasing runs of length $k$ (see e.g. [DB] and GJ, Exc. 4.2.8]). The connection between enumeration of permutations and sequences with forbidden subwords is also known (see [GJ, §4.2] for details and further references).

6.6. As we mentioned in the introduction, the most famous application of the MacMahon Master Theorem is to binomial identities [GJ]. It would be interesting to find applications of Theorem 2 in this direction.

\section{ACKNOWLEDGMENTS}

We are grateful to Roland Berger, Dominique Foata, Matjaž Konvalinka, Vic Reiner and Vladimir Retakh for interesting discussions and help with the references. We are especially thankful to Ira Gessel, David Jackson and Richard Stanley who kindly showed to us how combinatorial identities (9) and (16) can be derived.

\section{REFERENCES}

[B] R. Berger, Koszulity for nonquadratic algebras, J. Algebra 239 (2001), 705-734. MR 1832913 (2002d:16034)

[CF] P. Cartier and D. Foata, Problèmes combinatoires de commutation et réarrangements, Lecture Notes in Mathematics, No. 85, Springer, Berlin, 1969; available electronically at http://www.mat.univie.ac.at/ ${ }^{\sim}$ slc/books/cartfoa.html MR0239978(39:1332)

[DB] F. N. David and D. E. Barton, Combinatorial Chance, Hafner, New York, 1962. MR:0155371 (27:5305)

[FH1] D. Foata and G.-N. Han, A new proof of the Garoufalidis-Lê-Zeilberger quantum MacMahon master theorem, J. Algebra 307 (2007), no. 1, 424-431. MR2278064 (2008b:05008)

[FH2] D. Foata and G.-N. Han, Specializations and extensions of the quantum MacMahon Master Theorem, Linear Algebra Appl. 423 (2007), no. 2-3, 445-455. MR2312419|(2008c:05012)

[FZ] D. Foata and D. Zeilberger, Laguerre polynomials, weighted derangements, and positivity, SIAM J. Disc. Math. 1 (1988), no. 4, 425-433. MR968850 (89m:05008)

[Fr] R. Fröberg, Koszul Algebras, Lecture Notes in Pure and Appl. Math., 205, Dekker, New York, 1999, 337-350. MR 1767430 (2001i:16046)

[GLZ] S. Garoufalidis, T. Tq Lê and D. Zeilberger, The quantum MacMahon master theorem, Proc. Natl. Acad. Sci. USA 103 (2006), no. 38, 13928-13931. MR2270337

[GJ] I. P. Goulden and D. M. Jackson, Combinatorial Enumeration, John Wiley, New York, 1983. MR702512 (84m:05002)

[HL] P. H. Hai and M. Lorenz, Koszul algebras and the quantum MacMahon master theorem, Bull. Lond. Math. Soc. 39 (2007), no. 4, 667-676. MR2346948

[KP] M. Konvalinka and I. Pak, Non-commutative extensions of the MacMahon master theorem, Adv. Math. 216 (2007), no. 1, 29-61. MR2353248

[Ma] I. G. Macdonald, Symmetric Functions and Hall Polynomials, 2nd edition, The Clarendon Press, Oxford University Press, New York, 1995. MR 1354144 (96h:05207)

[MM] P. A. MacMahon, Combinatory Analysis, 2 vols., Cambridge University Press, 1915 and 1916; reprinted in one volume by Chelsea, New York, 1960. MR 0141605 (25:5003) 
[PP] A. Polishchuk and L. Positselski, Quadratic Algebras, University Lecture Series, 37, Amer. Math. Soc., Providence, RI, 2005. MR2177131 (2006f:16043)

[R] N. Roby, Séries de Poincaré des algèbres $h$-extérieures (in French), Bull. Sci. Math. (2) 95 (1971), 3-14. MR.0282861 (44:95)

[S] R. P. Stanley, Enumerative Combinatorics, vols. 1, 2, Cambridge Studies in Advanced Mathematics, 49, 62, Cambridge University Press, Cambridge, UK, 1997, 1999. MR 1442260 (98a:05001) MR:1676282 (2000k:05026)

Department of Mathematics, Massachusetts Institute of Technology, Cambridge, Massachusetts 02139

E-mail address: etingof@math.mit.edu

Department of Mathematics, Massachusetts Institute of Technology, Cambridge, MASSACHUSETTS 02139

E-mail address: pak@math.mit.edu 